\title{
The Weighted Arithmetic Mean-Geometric Mean Inequality is Equivalent to the Hölder Inequality
}

\author{
Yongtao $\mathrm{Li}^{1}$, Xian-Ming $\mathrm{Gu}^{2,3, *(1)}$ and Jianxing Zhao ${ }^{4}$ \\ 1 School of Mathematics and Statistics, Central South University, Changsha, Hunan 410083, China; \\ yongtao_li@csu.edu.cn \\ 2 School of Economic Mathematics/Institute of Mathematics, Southwestern University of Finance and \\ Economic, Chengdu, Sichuan 611130, China \\ 3 Johann Bernoulli Institute of Mathematics and Computer Science, University of Groningen, Nijenborgh 9, \\ P.O. Box 407, 9700 AK Groningen, The Netherlands \\ 4 College of Data Science and Information Engineering, Guizhou Minzu University, Guiyang, \\ Guizhou 550025, China; zhaojianxing@gzmu.edu.cn \\ * Correspondence: guxianming@live.cn or guxm@swufe.edu.cn or x.m.gu@rug.nl; Tel.: +86-157-5630-7980
}

Received: 1 August 2018; Accepted: 27 August 2018; Published: 4 September 2018

\begin{abstract}
In the current note, we investigate the mathematical relations among the weighted arithmetic mean-geometric mean (AM-GM) inequality, the Hölder inequality and the weighted power-mean inequality. Meanwhile, the proofs of mathematical equivalence among the weighted AM-GM inequality, the weighted power-mean inequality and the Hölder inequality are fully achieved. The new results are more generalized than those of previous studies.
\end{abstract}

Keywords: weighted AM-GM inequality; Hölder inequality; weighted power-mean inequality; L'Hospital's rule

\section{Introduction}

In the field of classical analysis, the weighted arithmetic mean-geometric mean (AM-GM) inequality (see e.g., [1], pp. 74-75) is often inferred from Jensen's inequality, which is a more generalized inequality compared to the AM-GM inequality; refer to, e.g., [1,2]. In addition, the Hölder inequality [2] found by Leonard James Rogers (1888) and discovered independently by Otto Hölder (1889) is a basic and indispensable inequality for studying integrals and $L^{p}$ spaces, and is also an extension of the Cauchy-Bunyakovsky-Schwarz (CBS) inequality [3]. The Hölder inequality is used to prove the Minkowski inequality, which is the triangle inequality in the space $L^{p}(\mu)[4,5]$. The weighted power mean (also known as the generalized mean) $M_{r}^{m}(a)$ for a sequence $a=\left(a_{1}, a_{2}, \ldots, a_{n}\right)$ is defined as $M_{r}^{m}(a)=\left(m_{1} a_{1}^{r}+m_{2} a_{2}^{r}+\cdots+m_{n} a_{n}^{r}\right)^{\frac{1}{r}}$, which is a family of functions for aggregating sets of numbers, and plays a vital role in analytical inequalities; see $[2,6]$ for instance.

In recent years, many researchers have been interested in studying the mathematical equivalence among some famous analytical inequalities, such as the Cauchy-Schwarz inequality, the Bernoulli inequality, the Wielandt inequality, and the Minkowski inequality; see [7-13] for details. Additionally, these studies note the relations among the weighted AM-GM inequality, the Hölder inequality, and the weighted power-mean inequality are still less clear, although one inequality is often helpful to prove another inequality [1,12]. Motivated by these aforementioned studies, in the present note, the mathematical equivalence among three such well-known inequalities is proved in detail; the result introduced in [14] is also extended.

The rest of the present note is organized as follows. In the next section, we will present the detailed proofs of mathematical equivalence among three celebrated mathematical inequalities. Finally, the paper ends with several concluding remarks in Section 3. 


\section{Main Results}

The weighted AM-GM inequality, the Hölder inequality, and the weighted power-mean inequality [1] (pp. 111-112, Theorem 10.5) are first reviewed and they are often related to each other. Then the results of mathematical equivalence among three such inequalities will be shown.

Weighted AM-GM Inequality. If $0 \leq c_{i} \in \mathbb{R}(i=1, \ldots, n)$ and $0 \leq \lambda_{i} \in \mathbb{R}(i=1, \ldots, n)$ such that $\sum_{i=1}^{n} \lambda_{i}=1$, then

$$
\prod_{k=1}^{n} c_{k}^{\lambda_{k}} \leq \sum_{k=1}^{n} \lambda_{k} c_{k}
$$

Hölder Inequality. If $0 \leq a_{i}, b_{i} \in \mathbb{R}(i=1, \ldots, n)$ and $p, q \in \mathbb{R}^{+}$such that $p^{-1}+q^{-1}=1$, then

$$
\sum_{k=1}^{n} a_{k} b_{k} \leq\left(\sum_{k=1}^{n} a_{k}^{p}\right)^{\frac{1}{p}}\left(\sum_{k=1}^{n} b_{k}^{q}\right)^{\frac{1}{q}} .
$$

Weighted Power-Mean Inequality. If $0 \leq c_{i}, \lambda_{i} \in \mathbb{R}(i=1, \ldots, n)$ such that $\sum_{i=1}^{n} \lambda_{i}=1$, and $r, s \in \mathbb{R}^{+}$ such that $r \leq s$, then

$$
\left(\sum_{k=1}^{n} \lambda_{k} c_{k}^{r}\right)^{\frac{1}{r}} \leq\left(\sum_{k=1}^{n} \lambda_{k} c_{k}^{s}\right)^{\frac{1}{s}}
$$

The word "equivalence" between two statements $\mathbf{A}$ and $\mathbf{B}$, by convention, is understood as follows: A implies B and B implies A. Two equivalent sentences have the same truth value. Thus, this note reveals a connection (in the sense of art) between these two well-known facts.

Theorem 1. The Hölder inequality is equivalent to the weighted AM-GM inequality.

Proof. To show that (2) implies (1), let $a_{k}=\left(\lambda_{k} c_{k}\right)^{\frac{1}{p}}, b_{k}=\left(\lambda_{k}\right)^{\frac{1}{q}}$ in (2) for all $k$; then

$$
\sum_{k=1}^{n}\left[\left(\lambda_{k} c_{k}\right)^{\frac{1}{p}}\left(\lambda_{k}\right)^{\frac{1}{q}}\right] \leq\left(\sum_{k=1}^{n} \lambda_{k} c_{k}\right)^{\frac{1}{p}}\left(\sum_{k=1}^{n} \lambda_{k}\right)^{\frac{1}{q}} .
$$

Since $p^{-1}+q^{-1}=1$ and $\lambda_{1}+\lambda_{2}+\cdots+\lambda_{n}=1$, the inequality (4) can be rewritten as:

$$
\sum_{k=1}^{n} \lambda_{k} c_{k} \geq\left(\sum_{k=1}^{n} \lambda_{k} c_{k}^{\frac{1}{p}}\right)^{p} .
$$

Now using the inequality (5) successively, it follows that

$$
\begin{aligned}
\sum_{k=1}^{n} \lambda_{k} c_{k} & \geq\left(\sum_{k=1}^{n} \lambda_{k} c_{k}^{\frac{1}{p}}\right)^{p} \geq\left(\sum_{k=1}^{n} \lambda_{k} c_{k}^{\frac{1}{p^{2}}}\right)^{p^{2}} \\
& \geq \cdots \geq\left(\sum_{k=1}^{n} \lambda_{k} c_{k}^{\frac{1}{p^{m}}}\right)^{p^{m}} \geq \cdots
\end{aligned}
$$

By L'Hospital's rule, it is easy to see that

$$
\lim _{x \rightarrow 0^{+}} \ln \left(\sum_{k=1}^{n} \lambda_{k} c_{k}^{x}\right) / x=\sum_{k=1}^{n} \lambda_{k} \ln c_{k} .
$$


Thus,

$$
\lim _{x \rightarrow 0^{+}}\left(\sum_{k=1}^{n} \lambda_{k} c_{k}^{x}\right)^{\frac{1}{x}}=\prod_{k=1}^{n} c_{k}^{\lambda_{k}} .
$$

Thus, in (6), we can pass to the limit by $m \rightarrow+\infty$, giving $\sum_{k=1}^{n} \lambda_{k} c_{k} \geq \prod_{k=1}^{n} c_{k}^{\lambda_{k}}$; hence, (2) implies (1). To show the converse, all that is needed is a special case of (1),

$$
\lambda_{1} c_{1}+\lambda_{2} c_{2} \geq c_{1}^{\lambda_{1}} c_{2}^{\lambda_{2}}
$$

Since $p^{-1}+q^{-1}=1$, and by (7), thus

$$
\frac{1}{p} a_{k}^{p} \sum_{k=1}^{n} b_{k}^{q}+\frac{1}{q} b_{k}^{q} \sum_{k=1}^{n} a_{k}^{p} \geq a_{k} b_{k}\left(\sum_{k=1}^{n} b_{k}^{q}\right)^{\frac{1}{p}}\left(\sum_{k=1}^{n} a_{k}^{p}\right)^{\frac{1}{q}} .
$$

Summing over $k=1,2, \ldots, n$, it follows that

$$
\sum_{k=1}^{n} a_{k}^{p} \sum_{k=1}^{n} b_{k}^{q} \geq \sum_{k=1}^{n} a_{k} b_{k}\left(\sum_{k=1}^{n} b_{k}^{q}\right)^{\frac{1}{p}}\left(\sum_{k=1}^{n} a_{k}^{p}\right)^{\frac{1}{q}} .
$$

Thus, (1) implies (2).

Remark 1. The CBS inequality (the AM-GM inequality) is the special case of the Hölder inequality (the weighted AM-GM inequality); therefore, Theorem 1 is a generalization of the result established in [14].

Theorem 2. The Hölder inequality is equivalent to the weighted power-mean inequality.

Proof. For $r \leq s$, let $p=\frac{s}{r} \geq 1$ and $c_{k}=d_{k}^{s}$ in (5) for $k=1, \ldots, n$, then

$$
\sum_{k=1}^{n} \lambda_{k} d_{k}^{s} \geq\left(\sum_{k=1}^{n} \lambda_{k} d_{k}^{r}\right)^{\frac{s}{r}}
$$

Thus, rewriting (8) as $\left(\sum_{k=1}^{n} \lambda_{k} d_{k}^{s}\right)^{\frac{1}{s}} \geq\left(\sum_{k=1}^{n} \lambda_{k} d_{k}^{r}\right)^{\frac{1}{r}}$. Now the task is to prove that (3) implies (2); let $\lambda_{k}=b_{k}^{q}\left(\sum_{k=1}^{n} b_{k}^{q}\right)^{-1}, d_{k}=a_{k} / b_{k}^{q-1}, r=1, s=p$ in (3), then

$$
\begin{aligned}
\sum_{k=1}^{n} a_{k} b_{k} & =\left(\sum_{k=1}^{n} b_{k}^{q}\right)\left(\sum_{k=1}^{n} \frac{b_{k}^{q}}{\sum_{k=1}^{n} b_{k}^{q}} \cdot \frac{a_{k}}{b_{k}^{q-1}}\right) \\
& \leq\left(\sum_{k=1}^{n} b_{k}^{q}\right)\left(\sum_{k=1}^{n} \frac{b_{k}^{q}}{\sum_{k=1}^{n} b_{k}^{q}} \cdot\left(\frac{a_{k}}{b_{k}^{q-1}}\right)^{p}\right)^{\frac{1}{p}} \\
& =\left(\sum_{k=1}^{n} a_{k}^{p}\right)^{\frac{1}{p}}\left(\sum_{k=1}^{n} b_{k}^{q}\right)^{\frac{1}{q}} \cdot \square
\end{aligned}
$$

Remark 2. Here, we can give another proof that (3) implies (2). Repeatedly using inequality (3), it follows that 


$$
\sum_{k=1}^{n} \lambda_{k} c_{k} \geq\left(\sum_{k=1}^{n} \lambda_{k} c_{k}^{\frac{1}{2}}\right)^{2} \geq\left(\sum_{k=1}^{n} \lambda_{k} c_{k}^{\frac{1}{3}}\right)^{3} \geq \cdots \geq\left(\sum_{k=1}^{n} \lambda_{k} c_{k}^{\frac{1}{n}}\right)^{n} \geq \cdots .
$$

By L'Hospital's rule, it is easy to see that

$$
\sum_{k=1}^{n} \lambda_{k} c_{k} \geq \prod_{k=1}^{n} c_{k}^{\lambda_{k}}
$$

By using analogous methods from Theorem 1, it can be proved that

$$
\sum_{k=1}^{n} a_{k}^{p} \sum_{k=1}^{n} b_{k}^{q} \geq \sum_{k=1}^{n} a_{k} b_{k}\left(\sum_{k=1}^{n} b_{k}^{q}\right)^{\frac{1}{p}}\left(\sum_{k=1}^{n} a_{k}^{p}\right)^{\frac{1}{q}} .
$$

Theorem 3. The weighted power-mean inequality is equivalent to the weighted AM-GM inequality.

Proof. To show that (1) implies (3), we merely exploit a special case of (1),

$$
a_{1}^{\lambda_{1}} a_{2}^{\lambda_{2}} \leq \lambda_{1} a_{1}+\lambda_{2} a_{2}
$$

Here, we define $U_{n}(a)=\lambda_{1} a_{1}^{s}+\lambda_{2} a_{2}^{s}+\cdots+\lambda_{n} a_{n}^{s}$; let $a_{1}=\lambda_{k} a_{k}^{s}\left(U_{n}(a)\right)^{-1}, a_{2}=\lambda_{k}$ and $\lambda_{1}=$ $\frac{r}{s}, \lambda_{2}=1-\frac{r}{s}$ in $(9)$, then

$$
\lambda_{k} a_{k}^{r}\left(U_{n}(a)\right)^{-\frac{r}{s}} \leq \frac{r}{s} \cdot \lambda_{k} a_{k}^{s}\left(U_{n}(a)\right)^{-1}+\left(1-\frac{r}{s}\right) \cdot \lambda_{k} .
$$

Summing over $k=1,2, \ldots, n$, then

$$
\sum_{k=1}^{n} \lambda_{k} a_{k}^{r}\left(U_{n}(a)\right)^{-\frac{r}{s}} \leq \sum_{k=1}^{n}\left[\frac{r}{s} \cdot \lambda_{k} a_{k}^{s}\left(U_{n}(a)\right)^{-1}+\left(1-\frac{r}{s}\right) \cdot \lambda_{k}\right]=1
$$

Therefore,

$$
\left(\sum_{k=1}^{n} \lambda_{k} c_{k}^{r}\right)^{\frac{1}{r}} \leq\left(\sum_{k=1}^{n} \lambda_{k} c_{k}^{s}\right)^{\frac{1}{s}}
$$

The converse is trivial from Remark 2.

\section{Concluding Remarks}

In this note, the mathematical equivalence among the weighted AM-GM inequality, the Hölder inequality, and the weighted power-mean inequality is investigated in detail. Moreover, the interesting conclusions of Lin's paper [14] are also extended. At the end of the present study, for convenience, the results on the equivalence of some well-known analytical inequalities can be summarized as follows:

- $\quad$ Equivalence of the Hölder's inequality and the Minkowski inequality; see [9].

- $\quad$ Equivalence of the Cauchy-Schwarz inequality and the Hölder's inequality; see [8].

- Equivalence of the Cauchy-Schwarz inequality and the Covariance-Variance inequality; see [7].

- Equivalence of the Kantorovich inequality and the Wielandt inequality; see e.g., [11]

- Equivalence of the AM-GM inequality and the Bernoulli inequality; see e.g., [10]

- Equivalence of the Hölder inequality and Artin's theorem; see e.g., [12] (pp. 657-663) for details.

- $\quad$ Equivalence of the Hölder inequality and the weighted AM-GM inequality; refer to Theorem 1.

- $\quad$ Equivalence of the Hölder inequality and the weighted power-mean inequality; see Theorem 2.

- Equivalence of the weighted power-mean inequality and the weighted AM-GM inequality; refer to Theorem 3. 
Author Contributions: All the authors inferred the main conclusions and approved the current version of this manuscript.

Funding: This work was funded by the National Natural Science Foundation of China (Grant No. 11501141), Science and Technology Top-notch Talents Support Project of Education Department of Guizhou Province (Grant No. QJHKYZ [2016]066).

Acknowledgments: The authors would like to thank Minghua Lin for his valuable discussions, which considerably improved the presentation of our manuscript.

Conflicts of Interest: The authors declare no conflict of interest.

\section{Abbreviations}

The following abbreviations are used in this manuscript:

AM-GM Arithmetic Mean-Geometric Mean

CBS Cauchy-Bunyakovsky-Schwarz

\section{References}

1. Cvetkovski, Z. Inequalities: Theorems, Techniques and Selected Problems; Springer: Berlin/Heidelberg, Germany, 2012; ISBN 978-3-642-23791-1.

2. Hardy, G.H.; Littlewood, J.E.; Pólya, G. Inequalities; Cambridge University Press: Cambridge, UK, 1934; ISBN 978-0-521-35880-4.

3. Dragomir, S.S. A Survey on Cauchy-Bunyakovsky-Schwarz Type Discrete Inequalities. J. Inequal. Pure Appl. Math. 2003, 4, 1-142. Available online: http://www.emis.de/journals/JIPAM/images/010_03_JIPAM/010_ 03.pdf (accessed on 5 April 2015).

4. Beckenbach, E.F.; Bellman, R. Inequalities; Springer: Berlin/Heidelberg, Germany, 1961; Volume 30, ISBN 978-3-642-64973-8.

5. Mitrinović, D.S. Analytic Inequalities; Springer: Berlin/Heidelberg, Germany, 1970; Volume 165, ISBN 978-3-642-99972-7.

6. Abramovich, S.; Mond, B.; Pečarić, J.E. Sharpening Jensen's inequality and a majorization theorem. J. Math. Anal. Appl. 1997, 214, 721-728. [CrossRef]

7. Fujii, M.; Furuta, T.; Nakamoto, R.; Takahashi, S.E. Operator inequalities and covariance in noncommutative probability. Math. Jpn. 1997, 46, 317-320.

8. Li, Y.-C.; Shaw, S.-Y. A Proof of Hölder Inequality Using the Cauchy-Schwarz Inequality. J. Inequal. Pure Appl. Math. 2006, 7, 1-3. Available online: http://www.emis.de/journals/JIPAM/images/299_05_JIPAM/ 299_05.pdf (accessed on 7 April 2015).

9. Maligranda, L. Equivalence of the Hölder-Rogers and Minkowski inequalities. Math. Inequal. Appl. 2001, 4, 203-207. [CrossRef]

10. Maligranda, L. The AM-GM inequality is equivalent to the Bernoulli inequality. Math. Intell. 2012, 34, 1-2. [CrossRef]

11. Zhang, F. Equivalence of the Wielandt inequality and the Kantorovich inequality. Linear Multilinear Algebra 2001, 48, 275-279. [CrossRef]

12. Marshall, A.W.; Olkin, I.; Arnold, B.C. Inequalities: Theory of Majorization and Its Applications, 2nd ed.; Springer Series in Statistics; Springer: New York, NY, USA, 2011; ISBN 978-0-387-40087-7.

13. Sitnik, S.M. Generalized Young and Cauchy-Bunyakowsky Inequalities with Applications: A survey. arXiv 2010, 1-51, arXiv:1012.3864. (In Russian)

14. Lin, M. The AM-GM inequality and CBS inequality are equivalent. Math. Intell. 2012, 34, 6. [CrossRef]

(C) 2018 by the authors. Licensee MDPI, Basel, Switzerland. This article is an open access article distributed under the terms and conditions of the Creative Commons Attribution (CC BY) license (http:/ / creativecommons.org/licenses/by/4.0/). 